\title{
Differences in airway wall compliance as a possible mechanism for wheezing disorders in infants
}

\author{
U. Frey*, A.C. Jackson**, M. Silverman*
}

Differences in airway wall compliance as a possible mechanism for wheezing disorders in infants. U. Frey, A.C. Jackson, M. Silverman. (CERS Journals Ltd 1998.

ABSTRACT: High-frequency input impedance measurements $(Z(f))$ provide useful noninvasive information on airway geometry and especially airway wall mechanics in the canine and human adult respiratory system. Using the high-speed interrupter technique (HIT), we have shown that it is possible to measure high-frequency $Z(f)$ in infants up to $900 \mathrm{~Hz}$, including antiresonant phenomena which are known to be related to wave propagation velocity. This implies that the first antiresonant frequency $(f a r, 1)$ is a function of airway wall compliance. Since, airway wall mechanics are particularly important for the flow limitation phenomena, we wondered whether we could find evidence that airway wall properties were important for the occurrence of flow limitation during incremental methacholine challenge in infants.

We measured $Z(f)$ from $32-900 \mathrm{~Hz}$ and maximal flow at functional residual capacity $\left(V^{\prime}\right.$ maxFRC) by the rapid chest compression technique in 10 infants (aged 36-81 weeks) with wheezing disorders.

far,1 increased significantly at very low doses of mch before any decline could be detected in $V^{\prime}$ maxFRC. We hypothesize that these changes in $f$ ar,1 are determined by mch-induced decrease in airway wall compliance.

High-speed interrupter technique in combination with rapid chest compression technique can be used to study developmental differences in airway function (particularly of airway wall properties) and their contribution to airway disease and response to bronchodilator therapy in infants.

Eur Respir J 1998; 12: 136-142.
*Dept of Child Health, University of Leicester School of Medicine, Leicester, UK. **Dept of Biomedical Engineering, Boston University, Boston, Massachusetts, USA.

Correspondence: U. Frey

Dept of Paediatrics

University Hospital of Bern

Inselspital

3010 Bern

Switzerland

Fax: 41316329468

Keywords: Airway wall forced oscillation technique interrupter technique respiratory impedance

respiratory mechanics wheezing

Received: March 251997

Accepted after revision March 131998

U. Frey was supported by a grant from the Swiss National Science Foundation (Stipendium für angehede Forscher der Forschungskommission der schweizerischen Nationalfonds) and a Swiss Sandoz Research Grant
The possibility that developmental differences in airway function might be risk factors for subsequent wheezing disorders in infancy has been systematically studied using, among other methods, the rapid chest compression technique (RTC) [1-5]. However, during flow limitation, maximum flows achieved during expiration are related directly to airway cross-sectional area and inversely to airway wall compliance [6]. The RTC cannot therefore distinguish between changes in airway calibre and airway wall properties, and developmental differences in airway wall properties rather than airway calibre [2-5] could be at least partly responsible for the occurrence of flow limitation in neonates and subsequent wheezing disorders in infants.

The hypothesis that airway wall properties might be particularly important in infants has also been raised by investigators who looked into bronchodilator responses in infants. PRENDIILLE et al. [7] found that in infants pretreat-ed with salbutamol, the maximal flow at functional residual capacity (FRC) (V'maxFRC) increased at low doses of methacholine but declined at higher doses. They specula-ted that at low doses, methacholine isometrically increased airway smooth muscle tone and facilitated wave propagation in the airways. This hypothesis has implications for understanding the potential adverse effects of bronchodilators on airway smooth muscle tone and therefore airway stability in the developing lung.
In order to facilitate the investigation of these issues, we need a noninvasive lung function technique that provides a measure of airway wall properties independently of airway calibre. We have introduced one such new method, the high-speed interrupter technique (HIT) [8], which enables the noninvasive and rapid measurement of high frequency input impedance $Z(f)$ in infants [9]. At highfrequencies $Z(f)$ contains antiresonances, that have been shown to be related to the propagation velocity of pressure waves within the airways (acoustic antiresonances) [9]. This implies that the frequency at which these antiresonances occur is dependent only on gas density, airway pathlength and airway wall properties [10]. High frequency $Z(f)$ potentially enables us therefore to measure airway wall properties noninvasively in infants for the first time.

The aim of the current study was to demonstrate that high-frequency $Z(f)$ values from $32-900 \mathrm{~Hz}$ were sensitive to changes in airway mechanics during methacholine induced airway obstruction in infants. Furthermore we aimed to demonstrate the importance of airway wall properties for flow limitation in infants by showing that the first antiresonance, as an indirect measure of airway wall compliance, changed at low doses of methacholine before there was any discernible reduction in $V^{\prime} \max -$ FRC. 


\section{Materials and methods}

\section{Study subjects}

The study was performed in 10 infants and young children (three females, seven males, aged 8-19 months) with a history of episodic or recurrent cough or wheeze, who had been referred from the outpatient clinic for lung function tests for clinical purposes (table 1). Two were premature infants with no chronic lung disease. Infants with other specific diseases and infants with upper respiratory tract infection within the previous 3 weeks were not included in the study. The infants and toddlers were sedated using a maximum oral dose of triclofos sodium of 150 $\mathrm{mg} \cdot \mathrm{kg}^{-1}$, and lung function was measured during behaviourally defined quiet sleep. The additional HIT measurements were approved by the Ethics Committee of the Royal Postgraduate Medical School, Hammersmith Hospital, London. Written consent was obtained from the parents.

\section{Study design}

In each infant, we performed 10 baseline measurements of $V^{\prime}$ maxFRC using the RCT, as well as 10 measurements of high-frequency $Z(f)$ by HIT. We then administered methacholine in doubling doses and performed five HIT and five RTC measurements after each dose until $V^{\prime}$ maxFRC had decreased by $>30 \%$. To demonstrate that HIT was sensitive to airway mechanics in individual subjects we compared baseline measurements with post-methacholine measurements of the frequencies $\left(f_{\mathrm{ar}}, 1, f_{\mathrm{ar}}, 2\right)$ and relative maxima in the real part $\left(Z(f) \mathrm{re}\left(f_{\mathrm{ar}, 1}\right), Z(f) \mathrm{re}\left(f_{\mathrm{ar}, 2}\right)\right)$ of the first and second antiresonances from the $Z(f)$ of individual subjects. To quantify the time course of effect on airway wall mechanics (e.g.far,1) in comparison with flow limitation ( $V^{\prime}$ maxFRC) during the methacholine challenge, we calculated the differences from baseline as standard deviation scores of each parameter derived from HIT and RTC at each provocation dose.

\section{Experimental protocol}

The principle and technical details of the HIT technique and rapid thoracic compression technique are described in the accompanying paper [9]. The infants were in the supine position. Transcutaneous oxtgen tension $\left(\mathrm{PO}_{2}\right)$ (TMC3, Radiometer, Copenhagen, Denmark) and transcutaneous oxygen saturation $\left(\mathrm{S}_{\mathrm{a}, \mathrm{O}_{2}}\right)$ (Biox 3740, Omeda, Omaha, NE, USA) were observed. The head position was standardized, based on the experience of DESAGER et al. [11], but

Table 1. - Physical characteristics of subjects

\begin{tabular}{lcccc}
\hline Group & $\begin{array}{c}\text { Weight } \\
\mathrm{kg}\end{array}$ & $\begin{array}{c}\text { Height } \\
\mathrm{cm}\end{array}$ & $\begin{array}{c}\text { GA } \\
\text { weeks }\end{array}$ & $\begin{array}{c}\text { PNA } \\
\text { weeks }\end{array}$ \\
\hline Mean & 10.7 & 78.6 & 38.8 & 61 \\
SD & 0.5 & 7.7 & 3.5 & 15.4 \\
Min & 8 & 70.2 & 30.0 & 36 \\
Max & 11 & 95.6 & 40.0 & 81 \\
\hline
\end{tabular}

GA: gestational age at birth; PNA: postnatal age. we did not tape the mouth for safety reasons. The head position was not changed between HIT- and RTC-measurements. The face mask was applied using a putty ring (Therapeutic Putty, Carter's, Bridgend, UK). The dead space in the face mask was reduced to $10-15 \mathrm{~mL}$, by filling it with putty.

At baseline, 10 sets of $Z(f)$ measurements were performed during quiet regular tidal breathing. Each measurement took $0.15 \mathrm{~s}$ at the beginning of inspiration. Impedance measurements with a coherence below 0.9 were not accepted. None of the infants was disturbed in sleep by the measurements. Thereafter, the inflatable jacket was loosely wrapped around the infant's chest and 10 forced expirations were performed.

The methacholine challenge test was performed as described previously [3] using cumulative doses of methacholine (saline, 0.5, 1, 2, 4, 8, 16, 32 and $64 \mathrm{~g} \cdot \mathrm{L}^{-1}$ ). At each provocation dose (PD), five sets of $Z(f)$ measurements (HIT) and then five RCT were performed, using the same jacket pressure throughout each test. The provocation test was terminated after a decrease of $V^{\prime} \operatorname{maxFRC}$ of $30 \%$ had been found. In one infant who showed a marked increase in $V^{\prime} \operatorname{maxFRC}$ after saline, PD was defined from the postsaline measurement.

Parameters extracted from $Z(f)$ features were the frequencies $(f a r, 1, f a r, 2)$ and the relative maxima in the real part $(Z(f)$ re $(f$ ar, 1$), Z(f)$ re $(f a r, 2))$ of the antiresonances defined by a zero crossing in the imaginary part and a relative maximum in the real part of the impedance spectrum. Since we are mainly interested in airway wall properties, we only focused on the description of these parameters and not on other parts of the $Z(f)$ spectrum. The significance of the change in $f$ ar, 1 and $Z(f) \operatorname{re}(f a r, 1)$ in individual subjects following methacholine challenge was determined by the t-test. For the whole group, differences in the values of $V^{\prime}$ maxFRC, $f$ ar, 1 and $Z(f)$ re $(f$ ar, 1$)$ before and after methacholine challenge were described using a paired $\mathrm{t}$ test, since the differences showed quasi-normal distributions.

\section{Standard deviation score analysis}

In order to investigate whether the frequency $f_{\text {ar }}, 1$ and the relative maximum in the real part $(Z(f) \operatorname{re}(f$ ar, 1$)$ of the first antiresonance changed at doses of methacholine lower than those which caused a detectable fall in $V^{\prime} \max -$ FRC, we expressed the change of $f_{\mathrm{ar}, 1} Z(f) \mathrm{re}\left(f_{\mathrm{ar}}, 1\right)$ and $V^{\prime}$ maxFRC at each PD by their change in standard deviations from the within-subject baseline mean. This statistical approach was valid because it took into account the technical variability of each parameter. Provocation dose levels were expressed as fractions $(1 / 8,1 / 4,1 / 2,1)$ of the final PD at which $V^{\prime}$ maxFRC had decreased by at least $30 \%$ from baseline.

\section{Results}

A representative example of $Z(f)$ at baseline and after methacholine challenge for one subject is shown in figure 1. Both the frequency $f$ ar, 1 of the first antiresonance as well as the relative maximum in the real part of $Z(f)$ at $f_{\text {ar, }} 1$ 

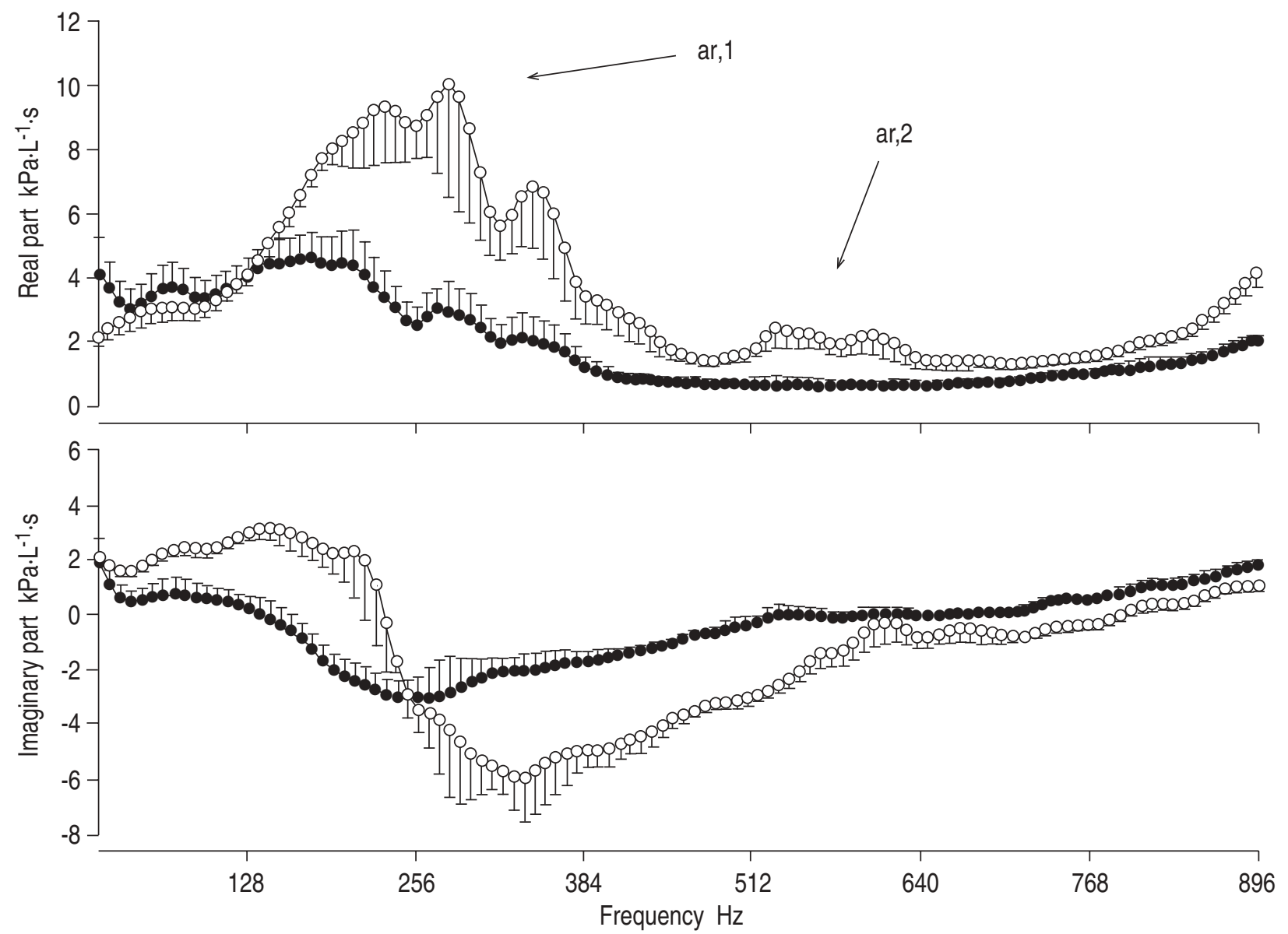

Fig. 1. - Representative example of the high-frequency impedance spectra before ( $\bullet$ : mean and SD of 10 measurements) and after methacholine challenge ( $O$ : mean and SD of five measurements) in subject. In this subject, the frequency $\left(f_{\text {ar }, 1}\right)$ and the relative maximum in the real part $\left(Z(f)\right.$ re $\left.\left(f_{\text {ar, }} 1\right)\right)$ of the first antiresonance $(\mathrm{ar}, 1)$ increased, and a second antiresonance $(\mathrm{ar}, 2)$ that had not been present at baseline developed after methacholine challenge at about $520 \mathrm{~Hz}$.

$(Z(f) \operatorname{re}(f a r, 1))$ increased following methacholine challenge (tables 2 and 3$)$. For the whole group of 10 infants, far, 1 and $Z(f)$ re $(f a r, 1)$ increased significantly $(\mathrm{p}=0.0025$ and $\mathrm{p}=0.005$, respectively) (fig. 2); $V^{\prime} \operatorname{maxFRC}$ decreased significantly $(\mathrm{p}<0.0005)$ confirming that a significant change in airway mechanics had been induced by the methacholine challenge. Considering individual responses, $V$ 'maxFRC decreased significantly in nine infants from the baseline value (exception: subject 8 , see below) and in all infants from the saline value. $f$ ar, 1 increased significantly in nine infants and $Z(f)$ re $(f a r, 1)$ increased significantly in eight of the infants (tables 2 and 3 ).

Although a second antiresonance was only found in five of 10 subjects at baseline, it was present in all subjects following methacholine challenge, leading to a relative maximum in the real part at about $600 \mathrm{~Hz}$ (range 554-

Table 2. - Baseline measurements

\begin{tabular}{|c|c|c|c|c|c|c|c|c|c|c|c|}
\hline & $\begin{array}{c}V^{\prime} \text { maxFRC } \\
\text { mean }\end{array}$ & $\begin{array}{c}V^{\prime} \operatorname{maxFRC} \\
\mathrm{CV}\end{array}$ & $\begin{array}{c}f_{\text {ar, } 1} \\
\text { mean }\end{array}$ & $\begin{array}{l}f_{\mathrm{ar}, 1} \\
\mathrm{CV}\end{array}$ & $\begin{array}{c}Z(f) \text { re }\left(f_{\text {ar }}, 1\right) \\
\text { mean }\end{array}$ & $\begin{array}{c}Z(f) \mathrm{re}\left(f_{\mathrm{ar}, 1}\right) \\
\mathrm{CV}\end{array}$ & $\begin{array}{c}f_{\mathrm{ar}, 2} \\
\text { mean }\end{array}$ & $\begin{array}{l}f_{\mathrm{ar}, 2} \\
\mathrm{CV}\end{array}$ & $\begin{array}{c}Z(f) \text { re }\left(f_{\text {ar }}, 2\right) \\
\text { mean }\end{array}$ & $\begin{array}{c}Z(f) \mathrm{re}\left(f_{\mathrm{ar}, 2}\right) \\
\mathrm{CV}\end{array}$ & $\begin{array}{c}\text { Ratio } \\
f_{\mathrm{ar}, 2} / f_{\mathrm{ar}, 1}\end{array}$ \\
\hline 1 & 146 & 5.1 & 117.5 & 3.3 & 2.6 & 7 & & & & & \\
\hline 2 & 320 & 4.9 & 162 & 6.7 & 5.3 & 8.8 & 554 & 6.3 & 1.8 & 30.9 & 3.4 \\
\hline 3 & 201 & 3.7 & 161 & 13.5 & 4.6 & 20.8 & & & & & \\
\hline 4 & 149 & 5 & 218.5 & 4.5 & 6.2 & 7.5 & & & & & \\
\hline 5 & 144 & 4.5 & 212.5 & 9.7 & 6.3 & 18.6 & 648 & 11.8 & 3.1 & 48.5 & 3.1 \\
\hline 6 & 409 & 5 & 177.9 & 14.1 & 6.4 & 8.9 & 540 & 6.4 & 2.3 & 26.8 & 3.0 \\
\hline 7 & 268 & 5.4 & 173 & 7.3 & 5.3 & 17 & & & & & \\
\hline 8 & 213 & 6 & 185.8 & 3.8 & 2.9 & 5.3 & & & & & \\
\hline 9 & 183 & 5.3 & 193 & 8.3 & 5.3 & 21.9 & & & & & \\
\hline 10 & 128 & 10.5 & 85.6 & 18.2 & 4.0 & 3.5 & 570 & 5.9 & 1.1 & 5.8 & 6.67 \\
\hline Mean & 216 & 5.5 & 168.7 & 8.9 & 4.9 & 11.9 & 578 & 7.6 & 2.1 & 28 & 3.2 \\
\hline SD & 91 & 1.8 & 40.8 & 4.9 & 1.4 & 6.9 & 48.2 & 2.8 & 0.8 & 17.5 & 0.2 \\
\hline
\end{tabular}

The input impedance $(Z(f))$ antiresonances were described by the frequencies $\left(f_{\mathrm{ar}, 1,}, \mathrm{far}_{2}(\mathrm{~Hz})\right)$ and the relative maxima in the real part at $f_{\text {ar, }, 1}$ and $f_{\text {ar, }}, 2$, respectively $\left(Z(f) \mathrm{re}\left(f_{\mathrm{ar}}, 1\right), Z(f) \mathrm{re}\left(f_{\mathrm{ar}, 2}\right)\left(\mathrm{kPa} \cdot \mathrm{L}^{-1} \cdot \mathrm{s}\right)\right)$. $V^{\prime} \operatorname{maxFRC}\left(\mathrm{mL} \cdot \mathrm{s}^{-1}\right)$ : maximal flow at functional residual capacity. The mean and coefficient of variation (CV) of 10 measurements are shown. 
Table 3. - Measurements following methacholine challenge

\begin{tabular}{|c|c|c|c|c|c|c|c|}
\hline $\begin{array}{l}\text { Subj. } \\
\text { no. }\end{array}$ & $\underset{\text { mean }}{f_{\mathrm{ar}}}$ & $\begin{array}{l}f_{\mathrm{ar}} \\
\mathrm{CV}\end{array}$ & $\underset{\mathrm{p} \text {-test }}{\mathrm{p}}$ & $\begin{array}{l}Z(f) \mathrm{re} \\
(f a r) \\
\text { mean }\end{array}$ & $\begin{array}{c}Z(f) r e \\
(f a r) \\
C V\end{array}$ & $\underset{\mathrm{t} \text {-test }}{\mathrm{p}}$ & $\begin{array}{c}\text { Ratio } \\
f_{\text {arr }, 2 /} \\
f_{\text {ar }, 1}\end{array}$ \\
\hline \multicolumn{8}{|c|}{ First antiresonance } \\
\hline 1 & 225.0 & 4.4 & $<0.001$ & 8.6 & 23.2 & $<0.001$ & \\
\hline 2 & 213.0 & 2.7 & $<0.001$ & 6.9 & 6.6 & $<0.05$ & \\
\hline 3 & 217.0 & 8.0 & $<0.005$ & 10.7 & 23.4 & $<0.001$ & \\
\hline 4 & 292.0 & 1.4 & $<0.001$ & 7.8 & 17.3 & $<0.01$ & \\
\hline 5 & 221.8 & 5.5 & NS & 7.4 & 8.1 & NS & \\
\hline 6 & 213.8 & 2.0 & $<0.01$ & 6.5 & 10.6 & NS & \\
\hline 7 & 257.6 & 10.5 & $<0.001$ & 10.9 & 25.5 & $<0.001$ & \\
\hline 8 & 201.2 & 11.1 & $<0.05$ & 6.4 & 10.9 & $<0.001$ & \\
\hline 9 & 218.6 & 4.0 & $<0.01$ & 6.9 & 6.7 & $<0.01$ & \\
\hline 10 & 124.3 & 6.8 & $<0.001$ & 4.6 & 5.3 & $<0.001$ & \\
\hline Mean & 218.4 & 5.6 & & 7.7 & 13.8 & & \\
\hline SD & 42.5 & 3.4 & & 2.0 & 7.9 & & \\
\hline \multicolumn{8}{|c|}{ Second antiresonance } \\
\hline 1 & 834.2 & 2.1 & & 1.7 & 7.7 & & 3.7 \\
\hline 2 & 596.0 & 0 & NS & 2.4 & 17.1 & NS & 2.8 \\
\hline 3 & 908.3 & 2.0 & & 4.9 & 3 & & 4.2 \\
\hline 4 & 854.3 & 6.2 & & 4.4 & 29.4 & & 2.9 \\
\hline 5 & 669.0 & 1.7 & NS & 2.7 & 10.3 & NS & 3 \\
\hline 6 & 554.0 & 6.4 & NS & 2.1 & 24.3 & NS & 2.6 \\
\hline 7 & 556.0 & 5.23 & & 2.4 & 20 & & 2.2 \\
\hline 8 & 532.0 & 0 & & 1.8 & 9.4 & & 2.6 \\
\hline 9 & 807.6 & 0.8 & & 2.8 & 9 & & 3.7 \\
\hline 10 & 612.0 & 1.1 & $<0.05$ & 1.0 & 21.2 & NS & 4.9 \\
\hline Mean & 692.3 & 2.6 & & 2.6 & 15.1 & & 3.1 \\
\hline SD & 143.7 & 2.5 & & 1.2 & 8.5 & & 0.6 \\
\hline
\end{tabular}

The input impedance $(Z(f))$ antiresonances were described by the antiresonant frequencies $\left(f_{\mathrm{ar}, 1}, f_{\mathrm{ar}, 2}(\mathrm{~Hz})\right)$ and the relative maxima in the real part at $f_{\mathrm{ar}}, 1$ and $f_{\mathrm{ar}, 2}$, respectively $\left(Z(f) \mathrm{re}\left(f_{\mathrm{ar},}, 1\right)\right.$, $\left.Z(f) \mathrm{re}\left(f_{\mathrm{ar}, 2}\right)\left(\mathrm{kPa} \cdot \mathrm{L}^{-1} \cdot \mathrm{s}\right)\right)$. The mean and coefficient of variation (CV) of 10 measurements are shown. Subj. no.: subject number.

$834 \mathrm{~Hz}$ ), but not a zero crossing in the imaginary part as in the well-defined first antiresonance. Following methacholine,

the frequency of this second antiresonance $(f a r, 2)$ occurring three times $f_{\mathrm{ar}, 1}$. $f_{\mathrm{ar}}, 2$ and $Z(f) \mathrm{re}\left(f_{\mathrm{ar}, 2}\right)$ did not significantly change in four of the five subjects in whom second antiresonances were detectable at baseline. One subject
(10) was different from the others. In this subject $f_{a r}, 2$ was found at a multiple of 7 of $f_{\mathrm{ar}}, 1$ at baseline, whereas following methacholine, $f_{\mathrm{ar}, 2}$ was five times $f_{\mathrm{ar}, 1}$. This led to a significant change in $f$ ar, 2 before and after methacholine challenge. However, we might not have been comparing corresponding antiresonances in this subject.

The averaged standard deviation scores at each provocation level for eight of the subjects are shown in figure 3. In these subjects, $V$ 'maxFRC did not change at low doses of methacholine $(1 / 8,1 / 4 \mathrm{PD})$ but increased suddenly and very rapidly at higher methacholine levels $(1 / 2 \mathrm{PD}, \mathrm{PD})$, whereas $f$ ar, 1 and $Z(f)$ re $\left(f_{\text {ar, }}, 1\right)$ already changed significantly at very low doses $(1 / 8$ PD) of methacholine, but then remained relatively constant. There was an intermediate change in $f$ ar, 1 and $Z(f)$ re $(f a r, 1)$ after saline inhalation.

Two infants (subjects 1 and 8) behaved exceptionally. In these subjects $V^{\prime}$ maxFRC significantly improved after saline and at low doses of methacholine, as shown by positive standard deviation scores (SDS) (fig. 4). In parallel to

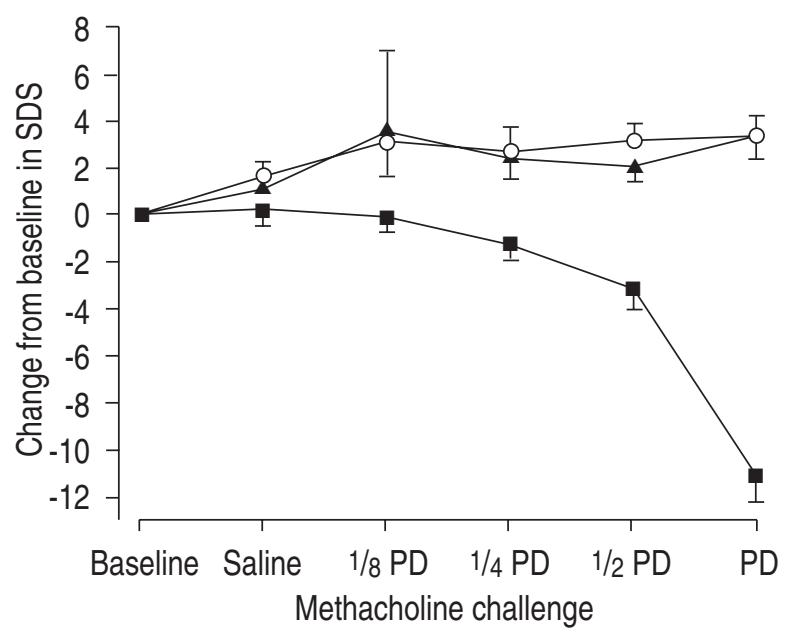

Fig. 2. - Standard deviation scores (SDS) calculated for each parameter (O: $f$ ar, $1 ; \mathbf{\Delta}: Z(f)$ re $(f a r, 1)$; $\left.\mathbf{\square}: V^{\prime} \operatorname{maxFRC}\right)$ at each provocation dose. In eight infants, these SDS were averaged ( $+\mathrm{SE}$ ) and plotted against the provocation doses of methacholine, expressed as a fraction of the provocation dose (PD) at which $V^{\prime}$ maxFRC had changed $>30 \%$. $f_{\text {ar, } 1}$ and $Z(f)$ re $\left(f_{\text {ar }, 1)}\right.$ changed significantly at much lower provocation doses than $V^{\prime} \operatorname{maxFRC}$. See legend to figure 1 for definitions.
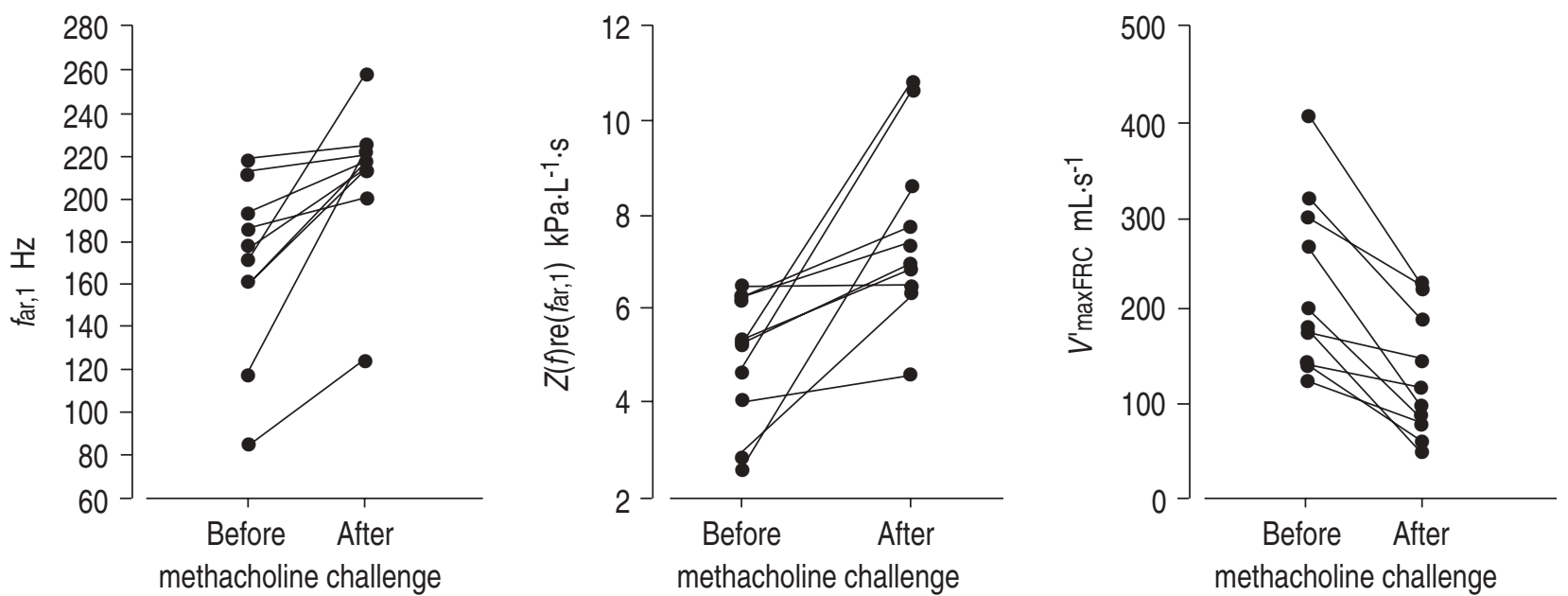

Fig. 3. - Graphs showing the significant (paired t-test: $\mathrm{p}<0.05)$ increase in frequency $\left(f_{\text {ar }, 1}\right)$ and relative maximum in the real part $(Z(f)$ re $(f a r, 1))$ of the first antiresonance following a possible methacholine challenge (as demonstrated by a decrease in maximal flow at functional residual capacity ( $V$ 'maxFRC) of $>30 \%$ in every subject). 

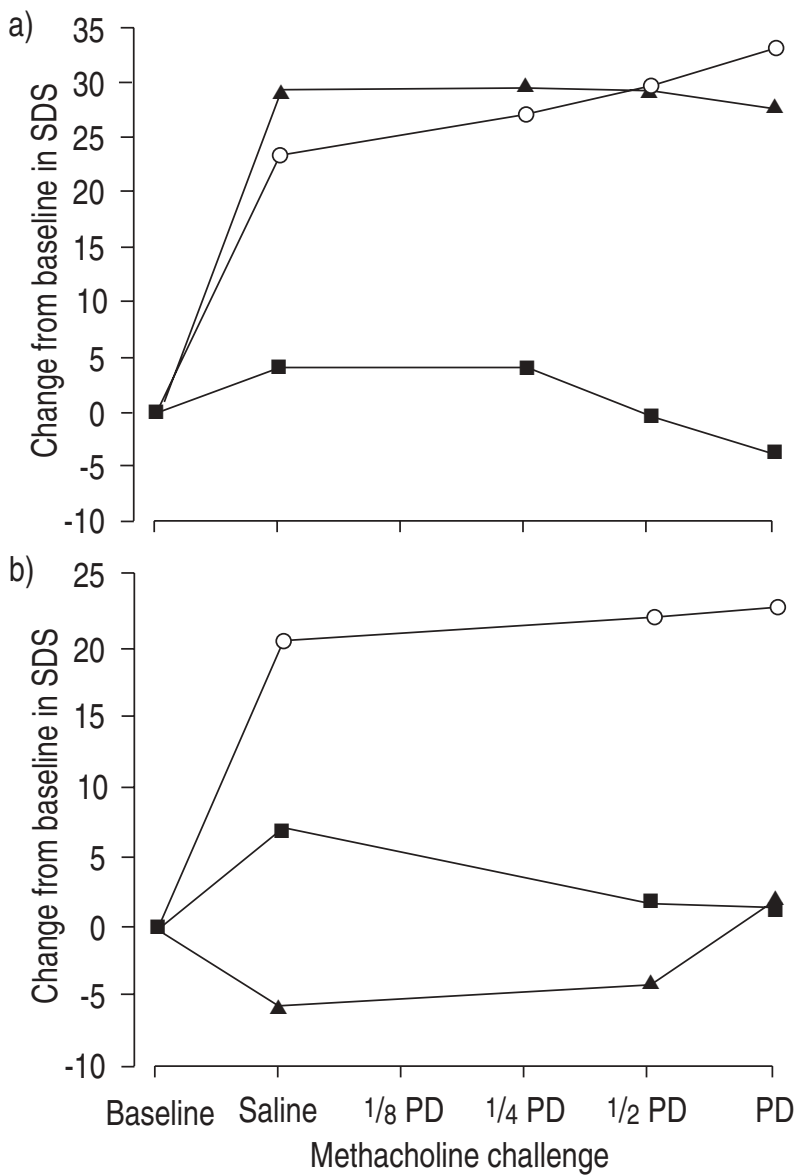

Fig. 4. - Standard deviation scores (SDS) calculated for each parameter (O: $f_{\text {arr }}, \mathbf{\Delta}: Z(f)$ re $\left(f_{\text {arr }} 1\right)$; $\mathbf{n}: V^{\prime}$ maxFRC $)$ at each provocation dose in: a) subject 1 ; and b) subject 8 . Both subjects ( 1 with episodic wheeze and 8 with wheeze shortly after birth following prematurity) showed an improvement in $V^{\prime}$ maxFRC (positive SDS) following saline and low doses of methacholine accompanied by an unusually large change in $f_{\mathrm{ar}, 1} 1$ and $Z(f)$ re $(f a r, 1)$. See legend to figure 1 for definitions.

this phenomenon $f_{\mathrm{ar}, 1}$ and $Z(f)$ re $(f a r, 1)$ changed several SDS at low doses of methacholine in these subjects. In subject 1 (fig. 4a), a term infant with episodic wheeze, this corresponded to an increase of far, 1 from $118-225 \mathrm{~Hz}$ $(190 \%)$ and $Z(f) \mathrm{re}(f \mathrm{ar}, 1)$ from $2.6-8.6 \mathrm{kPa} \cdot \mathrm{L}^{-1} \cdot \mathrm{s}(330 \%)$, indicating that this was not simply a result of a potentially small baseline SD. In the other subject (8) (fig. 4b), who had been born prematurely, $V$ 'maxFRC improved after saline then fell after methacholine. Similarly far,1 decreased initially after saline and then increased with increasing doses of methacholine, whereas $Z(f)$ re $(f a r, 1)$ increased following saline and low doses of methacholine by over $200 \%$.

\section{Discussion}

Developmental differences in airway function predispose to subsequent development of wheezing disorders in infancy [1-5]. Lower values of $V^{\prime}$ maxFRC in subsequently wheezing infants were interpreted as a result of smaller airway calibre [2-5]. However, maximal expiratory flow volume curves measured by the RTC technique cannot distinguish between the effects of airway calibre and airway wall properties on flow limitation. It might be especially important to distinguish these effects in infants since, as has been demonstrated in lambs by PANITCH et al. [12]), airway wall compliance is much higher than in later life. In order to understand the developmental basis for wheezing disorders, it is thus important to devise a technique to enable airway calibre and airway wall properties to be separately measured. We have addressed this problem by measuring airway mechanics during bronchial challenge with methacholine using the new noninvasive HIT $[8,9]$ in combination with the RTC. We found indirect evidence that airway wall properties changed during bronchoconstriction in infants, in such a way that the effects of airway wall compliance could be identified independently of those of airway calibre.

\section{High-frequency $Z(\mathrm{f})$ measurements in infants during methacholine challenge}

As previously shown (accompanying paper [9]) the frequencies of the antiresonances in the high-frequency $Z(f)$ spectra are related to wave propagation phenomena in the airways [9]. We found that $f$ ar, 1 and $Z(f) r e(f a r, 1)$ increased significantly in the group of 10 infants during methacholine challenge. Furthermore, we found that following methacholine challenge, a second antiresonance was detectable in all infants, whereas it was only present at baseline in five of the subjects. We have therefore shown that high frequency $Z(f)$ data are sensitive to changing airway mechanics.

Since the antiresonances have been shown to be related to wave propagation velocity in the airways, the frequency $f_{a r}, 1$ is a function only of mean airway pathlength and wave propagation velocity $[10,13]$. The latter is a function of gas density and airway wall compliance [10, 13]. If the changes in frequency had been due only to changes in airway pathlength, an increase of $200 \%$ in $f$ ar, 1 (as seen in some of the infants) would imply a shortening of the mean airway pathlength $50 \%$. It is, therefore, much more likely that the increase in far,1 was due to decreased airway wall compliance, probably due to a methacholine-induced increase in smooth muscle tone. A change in the airway wall compliance would cause an alteration in the propagation velocity of the pressure waves and hence change the first antiresonant frequency. We hypothesize that during methacholine challenge, airway wall properties and not simply airway calibre change.

Furthermore, in eight out of 10 subjects, standard deviation score analysis showed that far, 1 changed at much lower concentrations of methacholine than $V^{\prime}$ maxFRC but then remained relatively constant at higher concentrations while $V$ 'maxFRC continued to decrease. This would also support the hypothesis that the change in far,1 was mainly determined by changing airway wall properties rather than changing airway pathlength. If the pathlength had changed, it would probably be related to extreme narrowing or closure of the airways, in which case we would have expected a further increase of $f_{\mathrm{ar}, 1}$ with higher doses of methacholine, as well as a great change in $V^{\prime} \operatorname{maxFRC}$ at low doses of methacholine. Neither was observed. 


\section{Limits of the method}

The HIT technique was designed to give reliable data at high frequencies but not below $32 \mathrm{~Hz}$. Therefore, data are not directly comparable with those of previous authors. We have therefore focused only on $Z(f)$ data above 100 $\mathrm{Hz}$.

As in most lung function techniques in infants, the upper airways and the face mask influence physiological measurements. Whereas we have demonstrated in the accompanying paper [9] that adding a serial resistance to the upper airway did not change the antiresonant frequency, the influence of the compliance of the upper airways has to be discussed in more detail. The current data support the hypothesis that the antiresonances occurring above $100 \mathrm{~Hz}$ are related to wave propagation phenomena, implying that their frequencies are related to airway wall properties. We have to assume that the pressure waves propagate along the entire airway pathlength from the airway opening to the alveolar space. The compliance of the upper airway may, therefore, partly influence wave propagation velocity and the high-frequency impedance spectrum of the respiratory system. We have minimized the influence of the upper airway by filling the face mask with putty and stabilizing the cheeks by holding the face mask firmly in place. Since airway smooth muscle is the major determinant of intrathoracic airway wall compliance, and cholinergic receptors in the extrathoracic airway are only located in vessels and glands [14], we conclude that it is likely that intrathoracic airway compliance has changed during methacholine challenge. Nevertheless, we cannot completely rule out the possibility that upper airway compliance might also have changed to a minor degree, although the face-mask and the position of the upper airway were not altered during the challenge. There is a theoretical possibility that with increasing airway resistance during methacholine challenge, the pressure in the upper airways increased, which could lead to a secondary change in upper airway compliance. If true, one would expect changes in upper airway compliance at high doses of methacholine with considerable flow limitation. Our findings do not support this possibility, since the first antiresonance changed at low doses of methacholine when no measurable change in flow limitation was present.

The study was performed in infants with a history of wheezing disorders. The pathophysiology of wheezing disorders is heterogeneous. Whereas we expect the physiological mechanism of changing airway wall properties to occur in all infants, the effects on wave propagation might be variable in various groups of infants. The current data give a general idea of the possible mechanisms. However, in two infants, we observed different responses. Two ques-tions remain. Firstly, why did subject 8 (fig. 4 b) first decrease the antiresonant frequency at low doses of methacholine? To answer this question, we have to consider the frequency dependence of wave propagation velocity in a compliant tube [13]. Wave propagation velocity in a compliant tube depends on the frequency of the propagating pressure wave. This relationship is very complex and the effect of changing airway wall compliance on wave propagation velocity depends on the relationship between the frequency of the travelling pressure wave and the resonant frequency of the airway wall [13]. Theoretically the first antiresonant frequency could have decreased with decrea- sing airway wall compliance, if the airway wall resonance has been very different in this infant in comparison to the other infants. We saw a similar effect when we examined postocclusional pressure transients after flow interruption in school children [15].

The second question is: why did saline have an affect on $f_{a r}, 1$ in some infants? It has been pointed out that $0.9 \%$ saline is not "physiological". The $\mathrm{Na}^{+}$and $\mathrm{Cl}^{-}$content are $150 \mathrm{mmol} \cdot \mathrm{L}^{-1}$, and the $\mathrm{pH}$ is not 7 . Depending on the delivery device, the solution might become slightly hypertonic when administered. It could well be, therefore, that the HIT is sensitive enough to detect an effect of $0.9 \%$ saline on the airways. Another possibility that we have ruled out is the fact that the forced expiratory manoeuvres performed between baseline $Z(f)$ measurements and saline $Z(f)$ measurements could have altered the properties of the airways. We tested this hypothesis in four infants, in whom we performed 10 HIT measurements before and after $10 V^{\prime} \operatorname{maxFRC}$ manoeuvres at baseline. We found small differences in $Z(f)$ re $(f a r, 1)$, but they were not systematic, and we found no significant differences in $f a r, 1$.

The reason why $Z(f)$ re $(f a r, 1)$ increased during methacholine challenge is currently difficult to explain. Previous studies have shown that $Z(f)$ re $(f a r, 1)$ changed significantly during histamine challenge in tracheotomized dogs [15]. However, the situation in dogs is very different from the situation in infants where measurements have to be performed through the upper airways, including the face mask. Until detailed anatomical models of the infant bronchial tree are available that allow the development of distributed parameter models [16-18] to analyse high-frequency $Z(f)$, the change in $Z(f)$ re $(f a r, 1)$ has to considered as an empirical finding derived from a feature analysis.

\section{Hypothesis and implications for future research}

We hypothesize that at low doses of methacholine, the airways increase their smooth muscle tone, leading to a change in $f_{a r}, 1$ that potentially facilitates the propagation of flow in the airways. However, a minor degree of airway narrowing would inhibit flow propagation in the airways. The summed effect might result in no measurable change in $V^{\prime}$ maxFRC. At higher doses of methacholine, airway wall properties might change immeasurably (expressed by an plateau in $f$ ar, 1 ), but progressive airway narrowing leads to a fall in maximal flow. If this hypothesis is true, this has important physiological implications, because the quantitative effect of an unspecific bronchoconstricting agent will be determined by the state of airway wall compliance at baseline. We could think of two possible scenarios. Firstly, if the airway wall compliance at baseline is low, for instance, as a result of an increase in wall thickness caused by an chronic inflammatory process, the effect on calibre will be enhanced. Alternatively, if the airway wall compliance at baseline is very high (as shown in lambs [12]), the maximal flow might increase at low doses of a methacholine as the wall compliance decreases, stabilizing the airways and facilitating flow propagation in the airways. We saw the second scenario in two of the infants in our study, subject 8 who was born at 30 weeks gestational age, and in subject 1 , who wheezed only with upper respiratory tract infections (fig. 4). In these subjects $V^{\prime} \max -$ FRC improved following low doses of methacholine and 
subsequently decreased at higher doses of methacholine. In both subjects, we found that far, 1 changed much more than in the other infants, suggesting much greater changes in airway wall compliance at low doses of methacholine. Similar effects have been described by Prendiville et al. [7], who showed that in infants pretreated with salbutamol, which caused a relaxation of the airway smooth muscle and possibly an increase in airway wall compliance, $V^{\prime} \max -$ FRC improved at low doses of cumulative histamine and decreased only at higher concentrations.

\section{Summary}

We have shown that during bronchial challenge, highfrequency respiratory input impedance $Z(f)$ measured by the HIT is more sensitive to changes in airway mechanics than $V^{\prime}$ maxFRC at low doses of methacholine. With the high-speed interrupter technique, high-frequency $Z(f)$ data can be assessed noninvasively within seconds. Preliminary data show that these measurements can be made even in unsedated infants (unpublished observations). This makes the technique potentially very useful for clinical studies of changes in airway mechanics to monitor inhalation therapy as well as to assess bronchial hyperactivity in larger cohorts for research purposes.

Of particular interest is the fact that the first antiresonant frequency is likely to be largely a function of airway wall compliance. We found indirect evidence that airway wall compliance changes during bronchial challenge. This supports the hypothesis of Prendivile et al. [7], who showed that airway wall compliance has potentially an important role in flow limitation and wheezing in infants. We have to question, therefore, whether developmental differences in flow limitation in infants who subsequently develop wheezing disorders, are purely caused by smaller airway calibre or whether developmental differences in airway wall structure and, therefore, wall compliance might also be a possible mechanism that makes these infants more prone to develop wheezing disorders. Based on our findings, we proposed a hypothetical model to explain how airway wall properties and airway narrowing might interact during bronchial challenge. These airway wall effects might be important in infants with developmentally (premature infants) or acquired (inflammatory re-modelling) changes in airway wall compliance. They also may explain paradoxical effects of bronchodilators treatment in some infants, because these drugs not only cause bronchodilation but also remove airway smooth muscle tone, which is important for airway stability. High-frequency input impedance measurements might be useful in future research in these areas.

Acknowledgements: The authors thank A. Fritschi (Gambro AG, Switzerland) who designed the high-speed shutter valve based on our requirements and B. Suki (Dept of Biomedical Engineering, Boston University), who provided parts of the software to calculate respiratory input impedance (wave tube technique)

\section{References}

1. Tepper RS, Morgan WJ, Cota K, Wright A, Taussig LM. Physiologic growth and development of the lung during the first year of life. Am Rev Respir Dis 1986; 134: 513519.

2. Martinez F, Morgan W, Wright A, et al. Diminished lung function as a predisposing factor for wheezing respiratory illnesses in infants. $N$ Engl J Med 1988; 319: 1112-1117.

3. Clarke J, Reese A, Silverman M. Bronchial responsiveness and lung function in infants with lower respiratory tract illness in the first 6 months of life. Arch Dis Child 1992; 67: 1454-1458.

4. Tager IB, Hanrahan JP, Tosteson TD, et al. Lung function, pre- and post-natal smoke exposure, and wheezing in the first year of life. Am Rev Respir Dis 1993; 147: 811-817.

5. Brown RW, Hanrahan JP, Castile RG, Tager IB. Effect of maternal smoking during pregnancy on passive respiratory mechanics in early infancy. Ped Pulmonol 1995; 19: 23-28.

6. Dawson SV, Elliot EA. Wave speed limitation on expiratory flow - a unifying concept. J Appl Physiol 1977; 43: 498-515.

7. Prendiville A, Green S, Silverman M. Paradoxical response to nebulized salbutamol in wheezy infants, assessed by partial expiratory flow volume curves. Thorax 1987; 42: 86-91.

8. Frey U, Suki B, Kraemer R, Jackson AC. Human respiratory input impedance between 32 and $800 \mathrm{~Hz}$ measured by high speed interrupter technique. J Appl Physiol 1997; 82: 1018-1023.

9. Frey U, Jackson AC, Kraemer R, Silverman M. High frequency input impedance in infants assessed with the high speed interrupter technique. Eur Respir J 1998; 12: 148-158.

10. Jackson AC, Giurdanella CA, Dorkin HL. Density dependance of respiratory system impedances between 5 and 320 Hz in humans. J Appl Physiol 1989; 67: 2323-2330.

11. Desager KN, Buhr W, Willemen M, et al. Measurement of total respiratory impedance in infants by the forced oscillation technique. J Appl Physiol 1991; 71: 770-776.

12. Panitch HB, Deoras KS, Wolfson MR, Shaffer TH. Maturational changes in airway smooth muscle structure function relationship. Ped Res 1992; 31: 151-156.

13. Guelke RW, Bunn AW. Transmission line theory applied to sound wave propagation in tubes with compliant walls. Acustica 1981; 48: 101-106.

14. Baraniuk JN, Kaliner MA, Barnes PJ. Localisation of M3 muscarinic receptor messenger RNA in human nasal mucosa. Am J Rhinol 1992; 6: 145-148.

15. Frey U, Kraemer R. Oscillatory pressure transients after flow interruption during bronchial challenge test in children. Eur Respir J 1997; 10: 75-81.

16. Habib RH, Suki B, Bates JHT, Jackson AC. Serial distribution of airway mechanical properties in dogs: effect of histamine. J Appl Physiol 1994; 77: 554-566.

17. Jackson AC, Suki B, Ucar M, Habib R. Branching airway network models for analyzing high frequency lung input impedance. J Appl Physiol 1993; 75: 217-227.

18. Habib, RH, Chalker RB, Suki B, Jackson AC. Airway geometry and wall mechanical properties estimated from subglottal input impedance in humans. J Appl Physiol 1994; 77: 441-451. 